\title{
Fuzzy C-Means Algorithm-Based Adoption of Obturator Nerve Block under Adaptive Ultrasound Imaging for Bladder Tumor
}

\author{
Jianyun Hu $\mathbb{D}^{1},{ }^{1}$ Pinglin He $\mathbb{D}^{1},{ }^{1}$ Bixin Zhang $\mathbb{D}^{2},{ }^{2}$ Bin $S u\left(\mathbb{D},{ }^{2}\right.$ Jing Chen $\mathbb{D}^{3},{ }^{3}$ and \\ Haifeng $\mathrm{Hu}$ iD \\ ${ }^{1}$ Department of Urology, Affiliated Hospital of Chengdu University, Chengdu 610081, Sichuan, China \\ ${ }^{2}$ Department of Anesthesiology, Affiliated Hospital of Chengdu University, Chengdu 610081, Sichuan, China \\ ${ }^{3}$ Department of Ultrasound Medicine, Affiliated Hospital of Chengdu University, Chengdu 610081, Sichuan, China
}

Correspondence should be addressed to Haifeng Hu; 3115003014@m.fafu.edu.cn

Received 12 July 2021; Accepted 23 August 2021; Published 15 September 2021

Academic Editor: Gustavo Ramirez

Copyright (c) 2021 Jianyun Hu et al. This is an open access article distributed under the Creative Commons Attribution License, which permits unrestricted use, distribution, and reproduction in any medium, provided the original work is properly cited.

\begin{abstract}
This work aimed to study the adoption of obturator nerve block (ONB) based on adaptive medical ultrasound imaging under $\mathrm{C}$-means algorithm in transurethral resection of bladder tumor (TURBT). 120 patients with bladder tumors were diagnosed by C-means algorithm-based ultrasound imaging and were enrolled into group A (epidural anesthesia + resection), group B (general anesthesia), and group C (epidural anesthesia + ONB), each with 40 cases. The accuracy of the detection method, noise level, and complications before and after the operation were compared. All patients received TURBT for treatment. There was no significant difference in the general information of patients in each group $(P>0.05)$. As a result, the correct segmentation rate of the tumor region segmented by ultrasound imaging by C-means algorithm reached $95.6 \%$. The incidence of obturator nerve reflex (ONR) in group A $(7.5 \%)$ was greatly inferior to groups B and C $(P<0.05)$. The length of hospital stay in group A was $(4.01 \pm 1.43)$ days, which was notably different from groups $\mathrm{B}$ and $\mathrm{C}$, with considerable difference among the three $(P<0.05)$. In short, the adaptive medical ultrasound imaging under $\mathrm{C}$-means algorithm was more accurate in the diagnosis of bladder tumors. Moreover, ONB can effectively reduce the ONR and the incidence of complications in patients.
\end{abstract}

\section{Introduction}

With the continuous improvement of people's living standards, people's physical health problems have become the focus of attention. Bladder cancer is the most common urinary system tumor in China and Western countries. Appropriate initial resection plus accurate histological diagnosis of resected tumors is essential for the successful management of these tumors $[1,2]$. So far, transurethral resection of bladder tumor (TURBT) is still the most important treatment for bladder cancer [3]. In the current treatment of bladder tumors, the traditional treatment is through open surgery. This method alone is traumatic for patients and causes a lot of bleeding, and postoperative complications are also prone to occur [4]. With the continuous development of modern science and technology, resectoscope technology has been widely adopted, and TURBT has become the preferred surgical treatment for superficial bladder tumors $[5,6]$. The working principle of urethral resection technology refers to utilizing current to pass through the resection ring and locally producing high temperature vaporization or coagulation, so as to achieve the purpose of eliminating tumors [7]. This surgical method is less traumatic, causes less bleeding, and is less painful for the patient, and the patients can recover faster after surgery [8]. The difficulty of resection of bladder tumor is that the obturator nerve is close to the two inner walls of the bladder during surgical dissection. During the resection, the current will stimulate the obturator nerve through the bladder wall, which will cause the ONR. Suddenly, the adductor muscles of the thighs suddenly and strongly convulse and contract, which eventually leads to bladder perforation, pelvic vascular damage, nerve damage, and other serious complications [8]. Therefore, extra care should be given when resection of the bladder wall tumor is performed, so as to avoid ONR. This is also a key step for the success of the operation in the bladder tumor resection. 
To prevent or avoid ONR, medical experts and scholars have also adopted many treatment methods. They used general anesthesia muscle relaxants, epidural anesthesia muscle relaxants, or ONB anesthesia through nerve electrical stimulation until it was positioned. There was also the adoption of improved electric cutting methods or electric cutting devices, such as rapid and instant electric cutting, laser treatment, and changing the position of electrodes $[9,10]$. After research and experiments, it was found that, in all treatment methods, general anesthesia and muscle relaxants can prevent the occurrence of ONR. However, its disadvantages were that it had high requirements on doctors' skills and medical equipment and was prone to postoperative adverse reactions with high risks $[11,12]$.

Fuzzy C-means (FCM) clustering algorithm based on adaptive medicine is a classic algorithm among fuzzy clustering algorithms. It mainly utilizes the iterative optimization objective function to divide the data, and it has good convergence $[13,14]$. The research topic was mainly based on the ultrasonic image detection of bladder tumors under the fuzzy C-means algorithm and the comparison and analysis with radio-CT diagnosis. Through the adoption of different surgical methods, the feasibility and clinical effect of the TURBT ONB in bladder tumor resection based on fuzzy C-means algorithm were observed.

\section{Materials and Methods}

2.1. Selection of Research Samples. In this research, 120 patients with bladder tumors who were admitted to the hospital from January 20, 2018, to May 15, 2020, were selected for comparison between the diagnosis results of $\mathrm{C}$-means algorithm ultrasound imaging and radiological diagnosis. The accuracy of cystoscopy was set as the standard to judge its accuracy. Patients were enrolled into group A (epidural anesthesia + resection), group B (general anesthesia), and group $\mathrm{C}$ (epidural anesthesia $+\mathrm{ONB}$ ), each with 40 cases. All patients received TURBT for treatment. The research had been approved by the Medical Ethics Committee of the hospital, and the patients and their families understood the situation and signed the informed consent forms.

Inclusion criteria: diagnosis as bladder tumor by cystoscopy, CT, and so on; all patients were informed of the operation and signed informed consent form. Exclusion criteria: patients with severe cardiovascular disease, brain disease, and malignant tumor; patients with liver and kidney dysfunction; patients with metabolic disorders and drug allergies; patients with mental disorders; and patients in breast-feeding period.

2.2. Treatment between Groups. After successful epidural anesthesia in group A, a resection microscope was placed to observe the number and size of the tumor, and then the
ONB was blocked by transvesical puncture under the resection microscope. The puncture point was set about two transverse fingers above the pubic symphysis, and a disposable percutaneous renal puncture needle was adopted to insert the needle perpendicular to the skin into the bladder. Under the surveillance of the resectoscope, a needle was inserted into the mucosa around the tumor on the side wall of the bladder. After the bloodless needle was drawn back, about 3-5 mL of $2 \%$ lidocaine was injected into each point of the puncture needle, the puncture needle was then pulled out, and TURBT was performed routinely. After successful general anesthesia and muscle relaxant anesthesia in group B, TURBT was performed routinely. Group $C$ received epidural anesthesia and ONB located by nerve electrical stimulation device, and TURBT was performed routinely.

The judgement criteria were as follows.

The number of bladder tumors was calculated by the number of bladder wall tumors. Bladder tumor size was calculated based on the largest diameter of the bladder wall tumor. Obturator nerve reflex intensity: the lower limbs had no tremor, slight tremor, moderate shaking, and strong adduction as indicators of the ONR intensity. Successful ONB: no ONR or ONR occurred during the resection. ONB operation time was calculated from the time after the successful anesthesia to the beginning of the operation. The length of the patient's stay was calculated starting with the patient's hospitalization after surgery.

\subsection{Construction of Fuzzy C-Means Clustering Algorithm.} In this research, the fuzzy $\mathrm{C}$-means clustering algorithm introduces an adaptive weighting coefficient, which can automatically control the degree of influence of neighboring pixels, so it can accurately predict the gray value of the center pixel. The algorithm uses Fast Fuzzy C-Means (FFCM) to initially cluster the centers, so that its convergence speed can be greatly improved. The improved FCM algorithm requires a relatively large amount of calculation, so the neighborhood mean wave is introduced to modify the objective function. The equation is as follows:

$$
A_{s}=\sum_{k=1}^{e} \sum_{l=1}^{t} g_{k l}^{n}\left\|x_{l}-m_{k}\right\|^{2}+d \sum_{k=1}^{e} \sum_{l=1}^{t} g_{k l}^{n}\left\|\bar{x}_{l}-m_{k}\right\|^{2}
$$

where $d$ is the parameter that controls the smoothed image of the original image and the mean value of its corresponding neighborhood and $\bar{x}_{l}$ is the mean value of the neighborhood pixel at the center $x_{l}\left(\bar{x}_{l}\right.$ can be calculated in advance). This algorithm is denoted as FCM_Y1. When $d$ is 0 , the algorithm is equivalent to the standard FCM algorithm. When it tends to infinity, it is equivalent to using the standard FCM algorithm to segment the image after the mean is smoothed. 
When a similar standard FCM satisfies $\sum_{l=1}^{t} g_{k l}^{n}=1$, the necessary conditions for finding the minimum value of equation (1) are as follows:

$$
\begin{aligned}
& g_{k l}=\frac{\left\|x_{l}-m_{k}\right\|^{2}+d\left(\left\|\bar{x}_{l}-m_{k}\right\|^{2}\right)^{1 /(n-1)}}{\sum_{l=1}^{t}\left(\left\|x_{l}-m_{k}\right\|^{2}+d\left\|\bar{x}_{l}-m_{k}\right\|^{2}\right)^{1 /(n-1)}}, \\
& m_{k}=\frac{\sum_{l=1}^{t} g_{k l}^{n}\left(x_{l}+d \bar{x}_{l}\right)}{(1+d) \sum_{l=1}^{t} g_{k l}^{n}} .
\end{aligned}
$$

In general, when the gray average value of the center pixel is calculated, the influence of neighboring pixels on the center pixel is actually different. When there is a large difference between a certain pixel in the neighborhood and the center pixel, it is more likely that the neighborhood pixel is noise. To distinguish the different influences of neighboring pixels on the central pixel, adaptive weighted coefficient (AWC) is adopted in this research to filter the original image, which can reduce the loss of edge information and detail modes in the mean filtering and make full use of spatial information, which effectively improves the ability to suppress noise. The implementation of adaptive weighted mean filtering is described in Figure 1. follows:

The gray value equation of the image in the window is as

$$
p=\left\{y_{k} \mid y_{k} \in[0, M-1], \quad 1 \leq k \leq i * i\right\},
$$

where $y_{k}$ represents the gray value of the pixels in the window, the maximum gray level of the pixels in the window is recorded as $M$, and $G=i * i$ represents the total number of pixels in the window.

The window $H(K=1,2, \ldots, i * i)$ in the table is the adaptive weighting coefficient of the corresponding pixel in the template; it is determined by the following:

$$
H_{K}=\left(\frac{\left\|y_{k}-y_{i}\right\|^{2}}{\sum_{n=1}^{t_{o}}\left\|y_{n}-y_{i}\right\|^{2}}\right)^{-1},
$$

where $y_{i}$ is the center pixel, $y_{k}$ is its neighborhood pixel, $t_{o}$ is the total number of neighborhood pixels in the window, and $H_{K}$ corresponds to the adaptive weighting coefficient of the pixel. If the difference between $y_{k}$ and the center pixel $y_{i}$ is large, it indicates that the neighboring pixel is more likely to be noise, and the weighting coefficient of the center pixel should be as small as possible. If the difference between $y_{i}$ and the center pixel $y_{k}$ is small, it indicates that the neighboring pixel is more likely to be the original data, and its weighting coefficient for the center pixel should be as large as possible. This automatic control can be easily realized by equation (4).

The calculation equation for the adaptive weighted mean value of the center pixel in the window is as follows:

$$
\overline{y_{i}^{*}}=\frac{\sum_{n=1}^{t_{o}} H_{K} * y_{n}}{\sum_{n=1}^{t_{o}} y_{i}},
$$

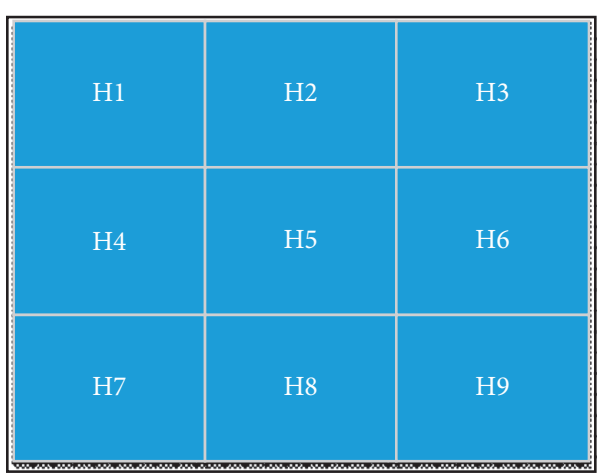

Figure 1: Weighting window.

where $\bar{y}_{i}^{*}$ is the adaptive weighted average gray value of the center pixel of the window. The modified objective function equation in this research topic is as follows:

$$
A_{s}=\sum_{k=1}^{e} \sum_{l=1}^{t} g_{k l}^{n}\left\|x_{l}-m_{k}\right\|^{2}+d \sum_{k=1}^{e} \sum_{l=1}^{t} g_{k l}^{n}\left\|y_{i}^{*}-m_{k}\right\|^{2},
$$

where $\bar{y}_{i}^{*}=(i=1,2, \ldots, n)$ is the pixel gray value in the adaptive weighted mean image. Under the condition of satisfying $\sum_{l=1}^{t} g_{k l}^{n}=1$, the minimization of equation (6) can obtain the iterative membership matrix and cluster centers.

$$
\begin{aligned}
& g_{k l}=\frac{\left\|x_{l}-m_{k}\right\|^{2}+d\left(\left\|y_{i}^{*}-m_{k}\right\|^{2}\right)^{-(1 /(n-1))}}{\sum_{k=1}^{e}\left(\left\|x_{l}-m_{k}\right\|^{2}+d\left(\left\|y_{i}^{*}-m_{k}\right\|\right)^{-(1 /(n-1))}\right.} \\
& m_{k}=\frac{\sum_{l=1}^{t} g_{k l}^{n}\left(y_{i}+d y_{i}^{*}\right)}{(1+d) \sum_{l=1}^{t} g_{k l}^{n}} .
\end{aligned}
$$

To quantitatively evaluate the performance of this method, the correct segmentation accuracy (SA) is introduced, which is calculated as follows:

$$
\mathrm{SA}=\frac{\mathrm{AC}}{\mathrm{T}} \times 100 \%,
$$

where AC represents the number of pixels that are correctly segmented and $T$ represents the number of all pixels. The correct segmentation rate of ultrasound imaging diagnosis and radiological diagnosis of the C-means algorithm is tested through experiments.

2.4. Statistical Methods. SPSS19.0 software was employed for data analysis. Mean \pm standard deviation $(\square x \pm s)$ was how measurement data were expressed, and percentage (\%) was how count data were expressed. One-way analysis of variance was adopted for pairwise comparison of running time. The comparison of age, height, weight, course of disease, male and female ratios, and diagnostic accuracy among groups was performed by paired $t$ test. The difference was statistically considerable at $P<0.05$. 


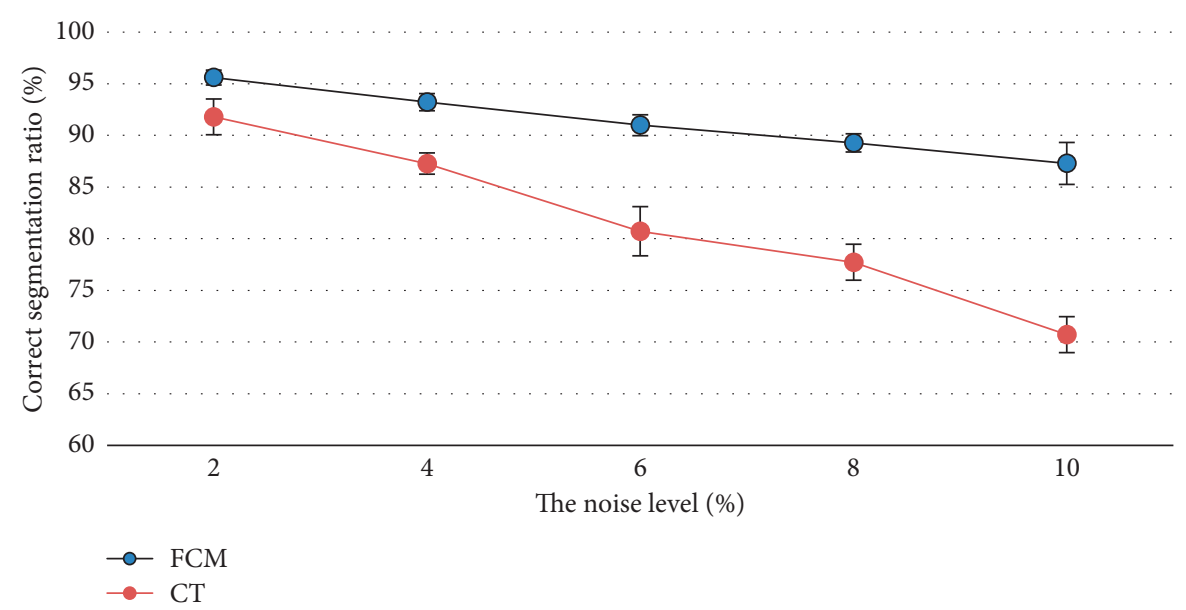

FIgure 2: The effect of different noise levels on the two detection methods.

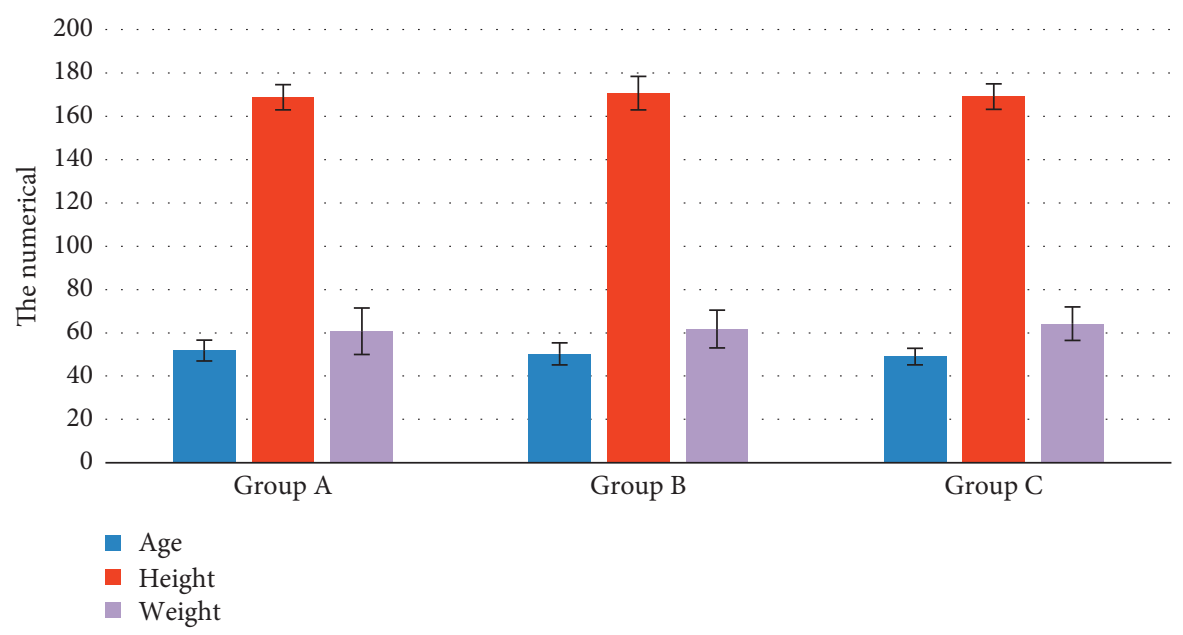

FIgURE 3: Comparison of basic patients' indicators.

\section{Results}

3.1. Comparison of Test Results of Two Test Methods. In this test, the two detection results were compared under different noise levels. The content of Gaussian noise in this research was $2 \%, 4 \%, 6 \%, 8 \%$, and $10 \%$, respectively. Figure 2 shows the comparison of the correct segmentation rates of the two detection methods. As the noise level continued to increase, the segmentation accuracy of the algorithm was constantly decreasing. However, when the influence of noise was small, the correct segmentation rate of the FCM algorithm can reach 95.6\%, and it showed a slow downward trend. In CT radiology diagnosis, the highest accuracy rate was $91.8 \%$, and the downward trend was also obvious. The results showed that the FCM algorithm had a higher correct segmentation rate for bladder tumors.

3.2. Basic Information of the Patients. Figures 3 and 4 show the comparison of basic indicators such as age, height, and weight of the three groups of patients. The age, height, weight, tumor number, and tumor size of the three groups of patients were not considerably different $(P>0.05)$.

The image below shows a patient with bladder tumor detected by FCM. The patient was a 51-year-old male patient who was diagnosed with gross hematuria for a week. There was slight discomfort in the lower abdomen, no frequent urination, urgency, pain, and no symptoms. Urine routine was as follows: occult blood $3+$, protein $1+$, red blood cells (649.52 cells/mirolitre), and high power field (HPF). Ultrasound findings: the bladder was well filled, and a cauliflower-like hypoechoic mass was detected on the right side of the bladder. It was connected with the bladder wall with a wide base and did not move with the change of body position. Strong echoes like calcification were seen on the surface of the tumor (Figure 5(a)). Color Doppler showed that there was a strip-shaped blood flow signal at the base of the tumor that entered the tumor. Spectral Doppler measured the arterial spectrum, the peak flow velocity was $25 \mathrm{~cm} / \mathrm{s}$, and the RI was 0.45 . There were no abnormalities in both kidneys and ureters (Figure 5(b)). 


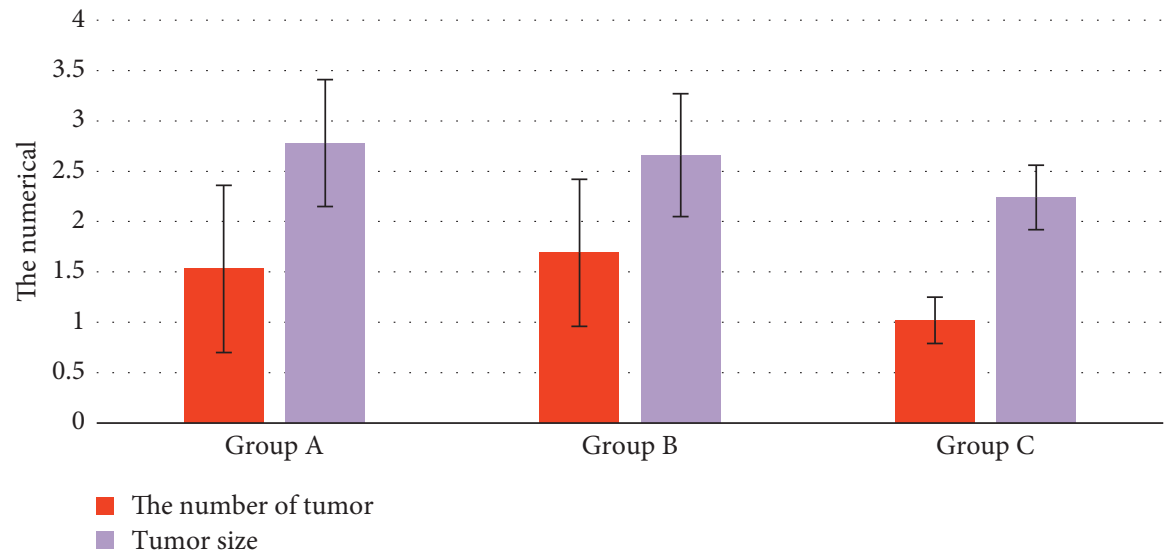

FIGURE 4: Comparison of pathological conditions of patients.

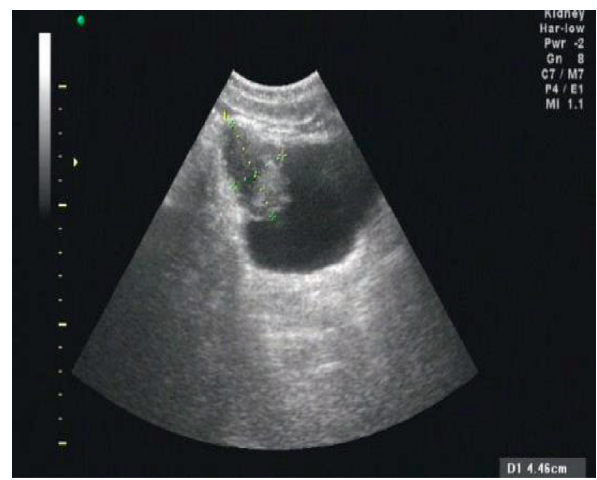

(a)

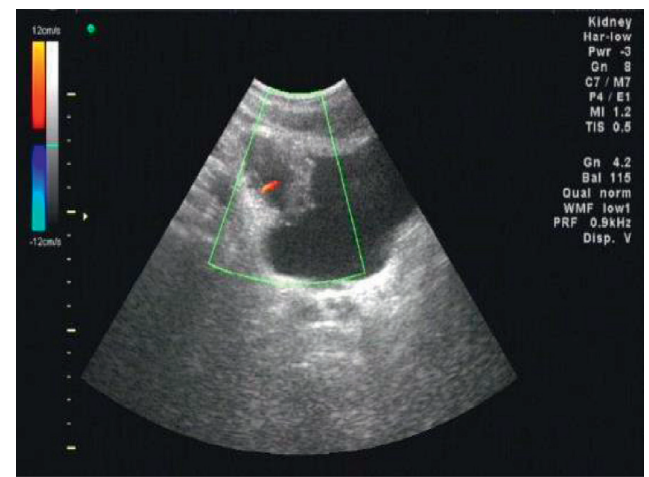

(b)

Figure 5: Color Doppler ultrasound test results of a male patient with bladder tumor.

3.3. The Incidence of ONR in Different Groups of Patients and Comparison of Complications before and after Surgery. From Figures 6 and 7, in the 40 cases of group A, after ONB, in TURBT, 37 cases of obturator nerves did not tremble, 1 case had slight tremor, 1 case had moderate tremor, and 1 case showed strong adduction. In one case, the obturator reflex disappeared after ONB anesthesia for the second time. One case was changed to general anesthesia after TURBT. The success rate of ONB reached $92.5 \%$ (Figure 8 ), and the incidence of ONR was $7.5 \%$. In group B, there were 30 cases of obturator nerve without tremor, 7 cases of slight tremor, 1 case of mild tremor, 1 case of moderate tremor, and 1 case of strong adduction. 4 cases of lung infection occurred, and the incidence of ONR was $25 \%$. In group $\mathrm{C}$, there were 24 cases of obturator nerve without tremor, 13 cases with slight tremor, 2 cases with moderate tremor, and 1 case with strong adduction. Wound bleeding occurred in 2 cases, urinary tract infection and bladder perforation occurred in 2 cases, and the incidence of ONR was $40 \%$.

In the results, the incidence of ONR in group A was greatly inferior to that in groups $\mathrm{B}$ and $\mathrm{C}$, with remarkable difference $(P<0.05)$. From Figure 9 , in the case of complications, the complication rate of group A was 0 , and the probability of lung infection in group B was obviously different than that of the other two groups $(P<0.05)$. The probability of urinary tract infection and bladder perforation in group $\mathrm{C}$ was higher than that of the other two groups. Moreover, the success rate of ONB in group A was also considerably higher than that in groups $\mathrm{B}$ and $\mathrm{C}$.

3.4. Comparison of ONB Operation Time of the Three Groups of Patients. Figure 8 shows that the ONB operation time of group A was $(4.09 \pm 1.38) \mathrm{min}$, the time of group B was $(8.99 \pm 2.73) \mathrm{min}$, and the time of group $\mathrm{C}$ was $(15.72 \pm 4.81)$ min. The time spent in group A was the least, which was remarkably different from group $\mathrm{B}$ and group $\mathrm{C}(P<0.05)$.

\subsection{Comparison of Hospitalization Days and Hospitalization} Expenses of the Three Groups of Patients. Figure 10 shows a comparison of the number of days in hospital after surgery for the three groups of patients. The length of hospital stay for patients with bladder tumor in group A was (4.01 \pm 1.43$)$ days, that in group B was $(10.93 \pm 2.82)$ days, and that in group $\mathrm{C}$ was $(7.02 \pm 3.92)$ days. Relative to group $\mathrm{B}$ and group $C$, group A had extremely shorter stay duration $(P<0.05)$.

Figure 11 shows the comparison of hospitalization expenses after surgery for the three groups of patients. The hospitalization 


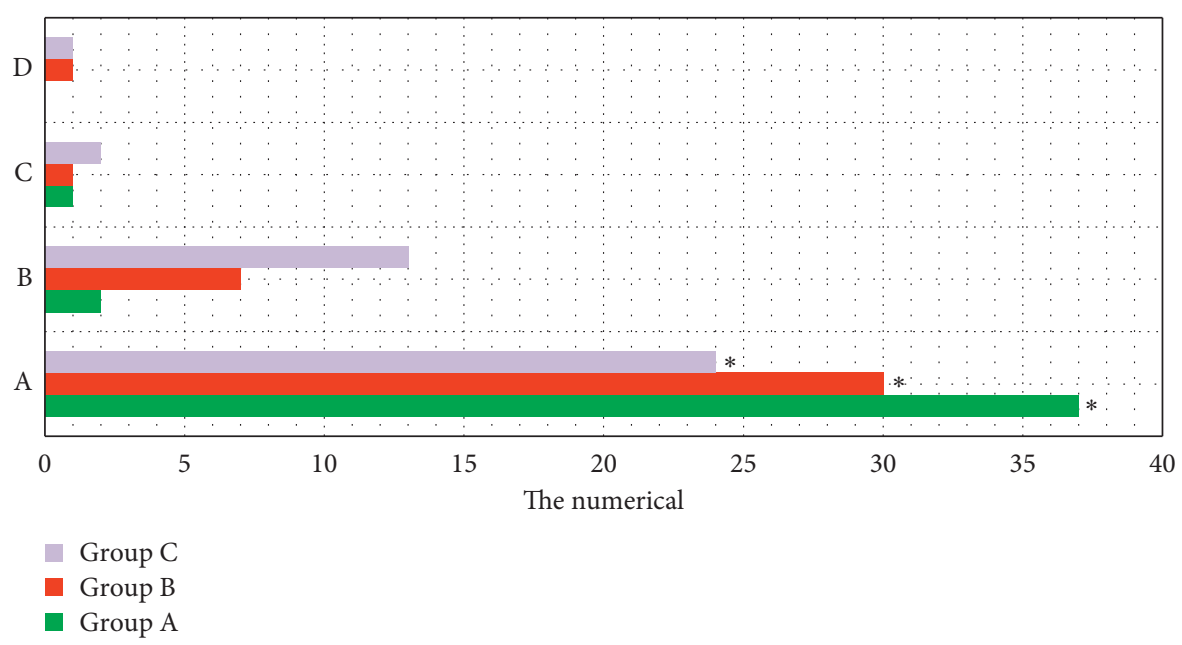

FIgURE 6: Obturator nerve reflex in the three groups. (a) Obturator nerve without tremor. (b) Slight tremor. (c) Moderate tremor. (d) Strong adduction. ${ }^{*}$ Indicates statistically significant difference $(P<0.05)$.

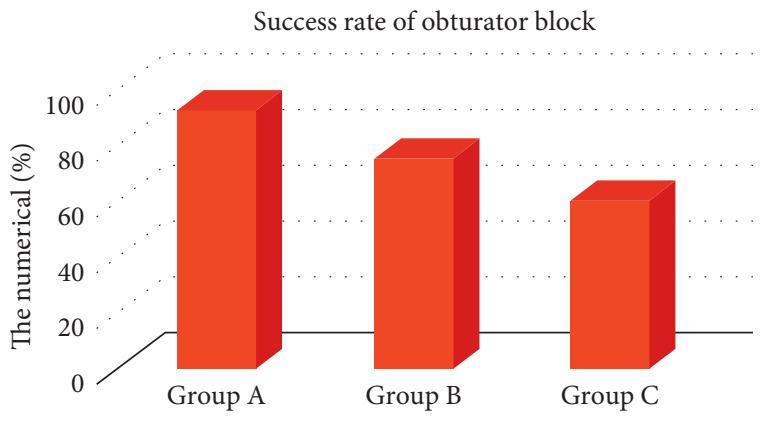

Figure 7: Comparison of ONB success rates among the three groups of patients.

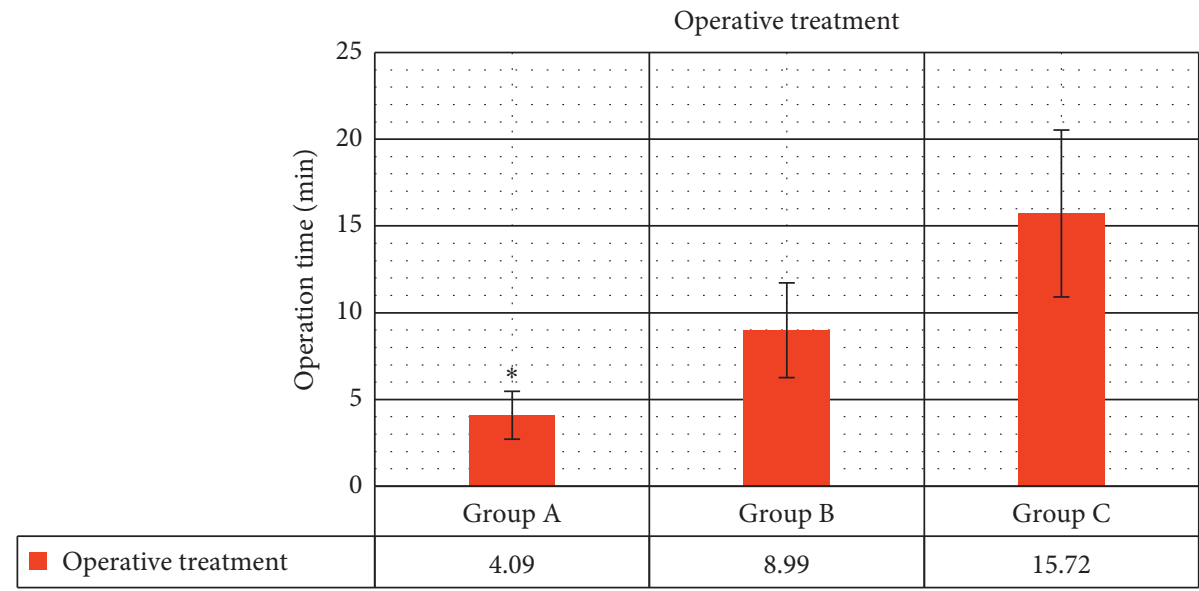

Figure 8: Comparison of the time spent on ONB operation in the three groups of patients. ${ }^{*}$ Indicates statistically significant difference $(P<0.05)$.

cost of bladder tumor patients in group A was $(5738.28 \pm 483.02)$ yuan, that of group B was $(9283.28 \pm 720.72)$ yuan, and that of group C was $(9982.73 \pm 993.73)$ yuan. Patients in group A were substantially different from those in groups B and $C$ regarding the expenses, and there were great differences among the three $(P<0.05)$.

\section{Discussion}

Bladder tumor is a very common tumor in urology in China. The incidence of urothelium is as high as $95 \%$ or more, and it has the characteristics of multicenter and recurrence [15]. Therefore, choosing an appropriate treatment plan has always 


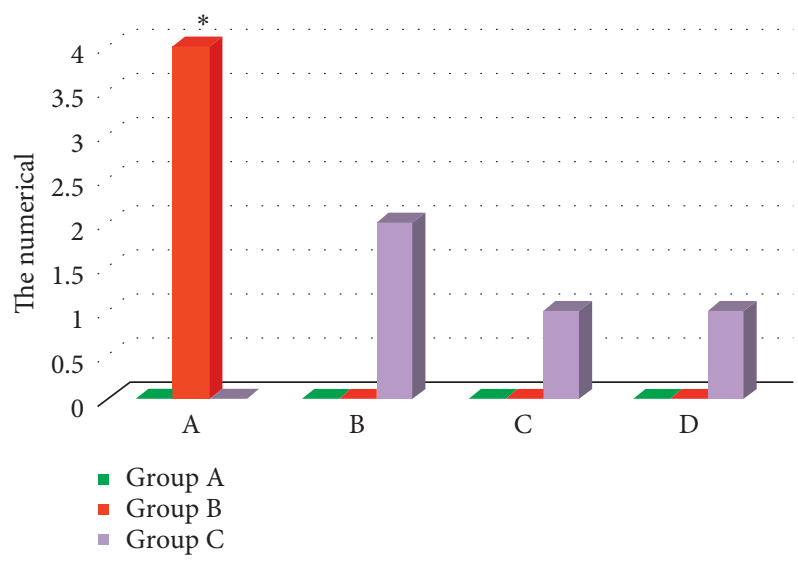

Figure 9: Comparison of complications among the three groups of patients. (a) Lung infection. (b) Wound bleeding. (c) Urinary tract infection. (d) Bladder perforation. ${ }^{*}$ Indicates statistically significant difference $(P<0.05)$.

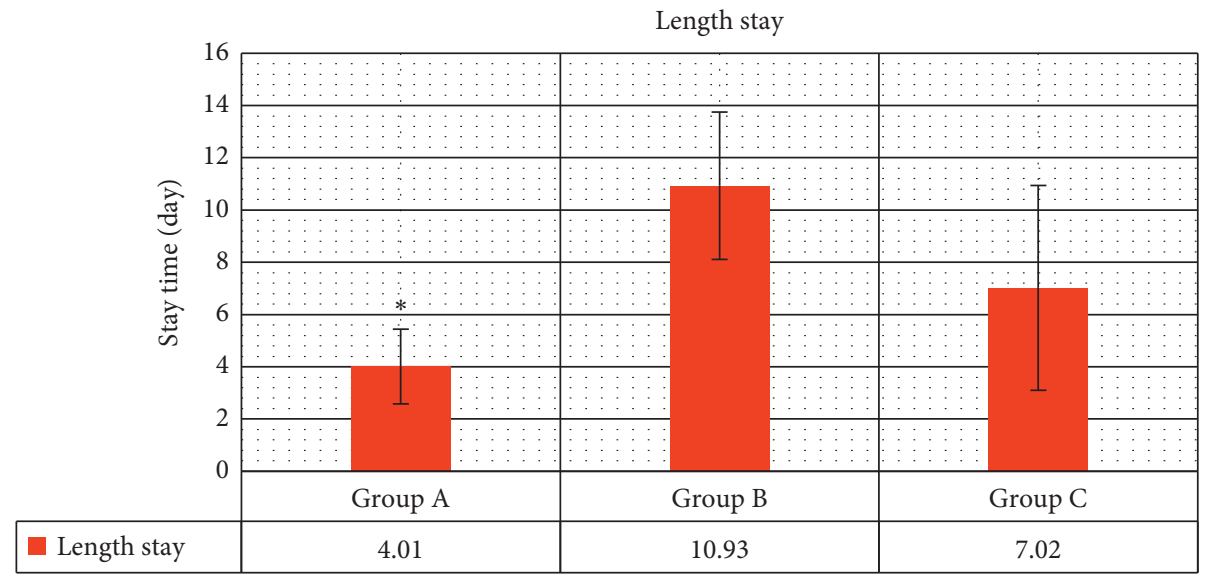

Figure 10: Comparison of the average length of stay in the three groups of patients. ${ }^{*}$ Indicates statistically significant difference $(P<0.05)$.

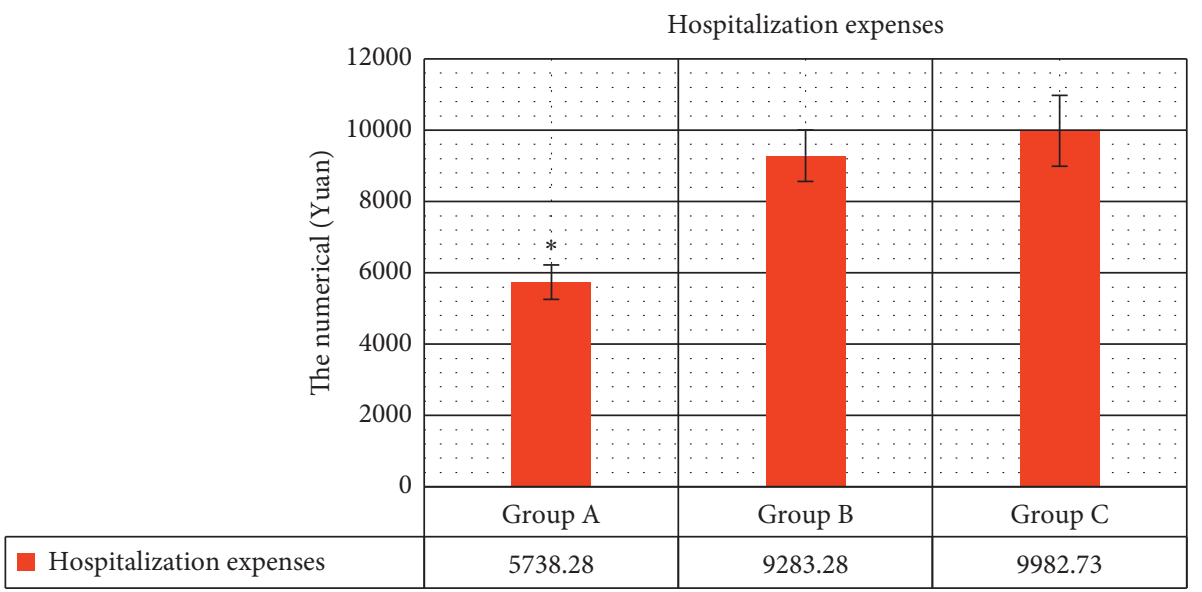

Figure 11: Comparison of hospitalization expenses of the three groups of patients. ${ }^{*}$ Indicates statistically significant difference $(P<0.05)$.

been a clinical difficulty. The fuzzy C-means clustering algorithm based on adaptive medicine is a classic algorithm among fuzzy clustering algorithms. It mainly uses iterative optimization of the objective function to perform fuzzy division of data and has good convergence. In this research, the improved fuzzy
C-means clustering algorithm introduced an adaptive weighting coefficient, which can automatically control the degree of influence of neighboring pixels. Therefore, the gray value of the center pixel was accurately estimated. The results showed that when the influence of noise was small, the correct segmentation 
rate of the FCM algorithm can reach $95.6 \%$, and it showed a slow downward trend. In CT radiological diagnosis, the highest accuracy rate was $91.8 \%$, and the downward trend was also obvious. This test showed that the FCM algorithm had a higher correct segmentation rate for bladder tumors, which was consistent with the results of Mishro et al. [16], proving that the diagnostic accuracy of the algorithm was high and it can be used clinically and widely promoted.

The algorithm was applied to the ultrasound image detection of patients with bladder tumors, and it was found that color Doppler showed that there was a strip-shaped blood flow signal at the base of the tumor that entered the tumor. Spectral Doppler measured the arterial spectrum, which was conducive to the use of ultrasound to predict the diagnosis of bladder tumor patients. After treatment with epidural anesthesia and TURBT, the incidence of ONR in patients of group A with bladder tumors was $7.5 \%$, the success rate of $\mathrm{ONB}$ was 92.5, and the probability of complications was 0 . Moreover, in contrast to group $\mathrm{B}$ and group $\mathrm{C}$, remarkable differences were found $(P<0.05)$. The results were consistent with the research conclusions of Şişik and Sert [17], which showed that TURBT guided by the fuzzy C-means clustering algorithm had a good surgical effect and can effectively avoid the probability of obturator reflex. The research results also showed that the ONB operation time of group A was $(4.09 \pm 1.38) \mathrm{min}$, and the time used was the least, which was greatly different versus other groups $(P<0.05)$. The hospitalization days and hospitalization expenses of the three groups of patients after surgery were compared. The hospitalization days of patients with bladder tumors in group A were $(4.01 \pm 1.43)$ days, and the hospitalization expenses were $(5738.28 \pm 483.02)$ yuan, which were both substantially different from groups $B$ and $\mathrm{C}$, and evident differences were suggested among the three groups $(P<0.05)$. It further demonstrated that this surgical method had better curative effect and was easier to implement.

\section{Conclusion}

The fuzzy C-means clustering algorithm was adopted in this research and compared with traditional radiological CT diagnosis. Then, it was applied to the TURBT treatment of bladder tumor patients guided by ultrasound images. It turned out that the FCM algorithm had high correct segmentation rate for bladder tumors. In addition, the TURBT treatment under this algorithm can effectively avoid the probability of ONR and increase the success rate of ONB. It also effectively reduced the occurrence of complications and can achieve the diagnosis and effective treatment of bladder tumor lesions. All in all, ONB can effectively reduce the ONR and the incidence of complications in patients, which was of high clinical promotion value and significance.

The disadvantage of this research is that the selected patient sample size is small and there is a certain deviation. The test time and equipment restrictions also have a certain impact on its results. In addition, the scope of application of the research results is also small. In subsequent studies, the sample size of patients will be increased and the scope of the research will be expanded to further explore the treatment of bladder tumor diseases. In short, the results of this article provide a certain reference for the treatment of bladder tumor.

\section{Data Availability}

The data used to support the findings of this study are available from the corresponding author upon request.

\section{Conflicts of Interest}

The authors declare that they have no conflicts of interest.

\section{References}

[1] S. Gurram, A. Muthigi, J. Egan, and L. Stamatakis, "Imaging in localized bladder cancer: can current diagnostic modalities provide accurate local tumor staging?" Current Urology Reports, vol. 20, no. 12, p. 82, 2019.

[2] B. Javanmard, M. R. Barghi, D. Amani, M. Fallah Karkan, and M. M. Mazloomfard, "Human papilloma virus DNA in tumor tissue and urine in different stage of bladder cancer," Urology Journal, vol. 16, no. 4, pp. 352-356, 2019.

[3] H. Yang, N. Wang, S. Han et al., "Comparison of the efficacy and feasibility of laser enucleation of bladder tumor versus transurethral resection of bladder tumor: a meta-analysis," Lasers in Medical Science, vol. 32, no. 9, pp. 2005-2012, 2017.

[4] Y. Allory, "Histoséminaire sur les tumeurs de vessie - Introduction. Le diagnostic des tumeurs de vessie, face aux nouveaux traitements et aux nouvelles classifications moléculaires," Annales de Pathologie, vol. 36, no. 6, pp. 371-372, 2016.

[5] K. A. Richards, N. D. Smith, and G. D. Steinberg, "The importance of transurethral resection of bladder tumor in the management of nonmuscle invasive bladder cancer: a systematic review of novel technologies," Journal of Urology, vol. 191, no. 6, pp. 1655-1664, 2014.

[6] D. Zainfeld and S. Daneshmand, "Transurethral resection of bladder tumors: improving quality through new techniques and technologies," Current Urology Reports, vol. 18, no. 5, p. 34, 2017.

[7] G. Mantica and C. Terrone, "Re: Eugene Shkolyar, Xiao Jia, Timothy C. Chang, et al. Augmented Bladder Tumor Detection Using Deep Learning. Eur Urol 2019;76:714-8," European Urology, vol. 76, pp. 714-718, 2019.

[8] W. S. Ham, W. T. Kim, H. J. Jeon, D. H. Lee, and Y. D. Choi, "Long-term outcome of simultaneous transurethral resection of bladder tumor and prostate in patients with nonmuscle invasive bladder tumor and bladder outlet obstruction," Journal of Urology, vol. 181, no. 4, pp. 1594-1599, 2009.

[9] Y. B. Kim, H. Y. Park, K. M. Kim, H. J. Shin, S. B. Kim, and M. G. Lee, "The effect of interfascial injection on obturator nerve block compared with nerve stimulating approach by ultrasound-guide: a randomized clinical trial," Urology Journal, vol. 16, no. 4, pp. 407-411, 201918.

[10] M. W. Kramer, J. J. Rassweiler, J. Klein et al., "En bloc resection of urothelium carcinoma of the bladder (EBRUC): a European multicenter study to compare safety, efficacy, and outcome of laser and electrical en bloc transurethral resection of bladder tumor," World Journal of Urology, vol. 33, no. 12, pp. 1937-1943, 2015.

[11] T. Takeuchi, A. Tonooka, Y. Okuno, M. Hattori-Kato, and K. Mikami, "Oct4B, CD90, and CD73 are upregulated in 
bladder tissue following electro-resection of the bladder," Journal of Stem Cells and Regenerative Medicine, vol. 12, no. 1, pp. 10-15, 2016.

[12] M. Brausi, B. Campo, G. Pizzocaro et al., "Intravesical electromotive administration of drugs for treatment of superficial bladder cancer: a comparative Phase II study," Urology, vol. 51, no. 3, pp. 506-509, 1998.

[13] J. H. Jung, A. Gudeloglu, H. Kiziloz et al., "Intravesical electromotive drug administration for non-muscle invasive bladder cancer," Cochrane Database of Systematic Reviews, vol. 9, no. 9, Article ID CD011864, 2017.

[14] J. Wang, S. Yu, C. Men et al., "Transurethral electric coagulation combined with retroperitoneal laparoscopic nephroureterectomy for upper urinary urothelial carcinoma," International Surgery, vol. 100, no. 3, pp. 547-551, 2015.

[15] H. Parastar and A. Bazrafshan, "Fuzzy C-means clustering for chromatographic fingerprints analysis: a gas chromatographymass spectrometry case study," Journal of Chromatography A, vol. 1438, pp. 236-243, 2016.

[16] P. K. Mishro, S. Agrawal, R. Panda, and A. Abraham, "A novel type-2 fuzzy C-means clustering for brain MR image segmentation," IEEE Transactions on Cybernetics, vol. 51, no. 8, pp. 3901-3912, 2021.

[17] F. Şişik and E. Sert, "Brain tumor segmentation approach based on the extreme learning machine and significantly fast and robust fuzzy C-means clustering algorithms running on Raspberry Pi hardware," Medical Hypotheses, vol. 136, Article ID 109507, 2020. 